\title{
Bioavailability differences in naltrexone: oral and injectable, extended release
}

\begin{abstract}
Undoubtedly, the rise of opioid use in the United States is a frightening upsurge. When exogenous opioids are ingested, they mimic the same pathways in the body that naturally-occurring morphine would. For this reason, if used in a manner that is not prescribed, opioids can be dangerous. Looking at opioid use disorder, several pharmacotherapies exist. One such pharmacotherapy that is studied in high volumes in the use of naltrexone, an opioid antagonist. This article looks at the significantly advantageous bioavailability factors of injectable, extended release naltrexone over daily, oral administration.
\end{abstract}

Keywords: naltrexone, bioavailability, injectable, extended release, oral, pharmacotherapies, opioid use disorder
Volume 3 Issue 6 - 2017

\section{Kristi N Lorelli}

Department of Pharmaceutical Sciences, St. John's University,

USA

Correspondence: Kristi N Lorelli, Department of Pharmaceutical Sciences, St. John's University, 8000 Utopia Parkway, Jamaica, NY, USA, Tel 602-677-369I,

Email Kristi.Lorellil7@my.stjohns.edu

Received: September 05, 2017 | Published: September 21 2017

\section{Introduction}

As of the present day, several forms of naltrexone exist, and injectable, extended release naltrexone is of great discussion in the addiction community. But since the bioavailability factors of oral naltrexone differ so significantly than injectable naltrexone, the latter is becoming increasingly studied in terms of adherence. The adherence statistics, though, not the pharmacokinetic factors of naltrexone, give credence to why most Americans still addictively consume opioids. Adherence to pharmacotherapies will naturally call upon a behavioral or a psychosocial component that this review will not explore at this time. Rather, this review will look at the bioavailability factors of oral naltrexone and compare them with the same factors of injectable, extended release naltrexone.

\section{Discussion}

In 1984, when oral naltrexone became federally approved by the United States Food and Drug Administration for the treatment of opioid dependence, its efficacy became known and broadly studied. Despite its impressible presence as a concomitant treatment additive, oral naltrexone, still could not curtail America's opioid epidemic. ${ }^{1,2}$ When, in 2016, the Substance Abuse and Mental Health Services Administration (SAMHSA) met to discuss the use of pharmacotherapies for opioid addiction, a harrowing statistic loomed over the members in presence. The United States, although, meek in terms of worlds' population, outweighed the worlds' consummation of opioid medications by $56 \% .^{3}$

One of the commonly used forms of opioid pharmacotherapy is naltrexone. ${ }^{4}$ Naltrexone is a pure opioidantagonistand unlikemethadone or buprenorphine, the patient must be completely detoxified from the opioid. The reasoning for this is based on neurobiology of addiction and the pharmacology of naltrexone. Regarding the neurobiology of opioid use disorder, when a person has pain, a naturally occurring pain reliever becomes released in the body; morphine. Morphine produces generalized CNS depression and therefore, will reduce painful stimuli. ${ }^{5}$ The way morphine is able to reduce painful stimuli is that within the body are receptors that are specific for opioids, such as naturally-occurring and even synthetic derivatives of morphine. ${ }^{6}$ These receptors, when engaged by an endogenous or exogenous drug, can either fully inhibit, disinhibit or partially inhibit the pathway for pain and reward. This cross interference between pain and reward is of interest to addiction specialists as, if overused, can lead to opioid use disorder. When looking at the pharmacology of naltrexone, a pure antagonist of opioid receptors, opioids cannot be present within the patient. Naltrexone attaches to opioid receptors and inhibit analgesic effects within the system. ${ }^{6}$ Once naltrexone enters the detoxified system, the drug binds and produces a blockade to opioid receptors when an opioid then becomes ingested. ${ }^{7}$ Maintenance of use of opioid antagonists such as naltrexone are widely studied as a part of opioid use disorder. Naltrexone is studied in high volumes because it can have the effect of attenuating the physiological craving that occurs within opioid use disorder and in turn could lessen the number of individuals affected. Looking specifically at oral naltrexone, it is quickly absorbed and has a half-life of $10.3 \pm 3.3$ to $9.7 \pm 1.1$ leading to a bioavailability of less than $50 \%{ }^{1,8}$

While within this half-life of oral naltrexone, peak plasma levels are reach at roughly four hours following administration. ${ }^{1}$ First pass metabolism for oral naltrexone occurs extensively in the liver, and is excreted via the renal system. Oral naltrexone, as stated, has a variable peak plasma parameter that can be of issue when exploring long-lasting effects within the patient. ${ }^{9}$ In comparison, an injectable, extended release formulation of naltrexone has been shown to correct for the variable peaks of plasma that oral naltrexone exhibits such a formulation will, presumably, occupy more mu opioid receptors and have a greater, more positive treatment outcome than by oral administration only. ${ }^{10}$ When injectable, extended release naltrexone is administered, plasma concentrations with 24 hours to three days of the injection, did not vary considerably throughout the course of detection. ${ }^{11}$ Injectable, extended release naltrexone has the steady state factor of being able to be kept within significant bioavailable parameters longer and less inconstant than does daily, oral administration of naltrexone.

\section{Conclusion}

In summation, although when approved for opioid depended in 1984, daily, oral naltrexone seemed to give practitioners and clients alike the hope in battling with opioid use disorders. Unfortunately, due to the bioavailability factors such as half-life, peak plasma rates, 
and over steady state of naltrexone, injectable, extended release naltrexone is far superior for the use of opioid use disorder.

\section{Acknowledgements}

None.

\section{Conflict of interest}

The author declares no conflict of interest.

\section{References}

1. Bart G. Maintenance medication for opiate addiction: the foundation of recovery. J Addict Dis. 2012;31(3):207-225.

2. Brooks AC, Comer SD, Sullivan MA, et al. Long-acting injectable versus oral naltrexone maintenance therapy with psychosocial intervention for heroin dependence: a quasi-experiment. $J$ Clin Psychiatry. 2010;71(10):1371-1378.

3. Chapman CR. Opioid Pharmacotherapy for chronic noncancer pain: the american experience. Korean J Pain. 2013;26(1):3-13.

4. Stotts AL, Dodrill CL, Kosten TR. Opioid dependence treatment: options in pharmacotherapy. Expert Opin Pharmacother. 2009;10(11):17271740 .
5. Stefano GB, Goumon Y, Casares F, et al. Endogenous morphine. Trends Neurosci. 2000;23(9):436-442.

6. Basbaum AI, Fields HL. Endogenous pain control mechanisms: review and hypothesis. Ann Neurol. 1978;4(5):451-462.

7. Kosten TR, Haile CN. Opioid-Related Disorders. In: Kasper D, Fauci A, et al. editors. Harrison's Principles of Internal Medicine. 19e. USA: New York, Mc Graw-Hill; 2014.

8. Littleton J, Zieglgänsberger W. Pharmacological mechanisms of naltrexone and acamprosate in the prevention of relapse in alcohol dependence. Am J Addict. 2003;12(Suppl 1):S3-S11.

9. Verebey K, Volavka J, Mulé SJ, et al. Naltrexone: disposition, metabolism, and effects after acute and chronic dosing. Clin Pharmacol Ther. 1976;20(3):315-328

10. Incorporating Alcohol Pharmacotherapies Into Medical Practice. Rockville (MD): Substance Abuse and Mental Health Services Administration (US), (Treatment Improvement Protocol (TIP) Series, No. 49.) Chapter 4-Oral Naltrexone. 2009.

11. Bartus RT, Emerich DF, Hotz J, et al. Vivitrex ${ }^{\circledR}$, an injectable, extended-release formulation of naltrexone, provides pharmacokinetic and pharmacodynamic evidence of efficacy for 1 month in rats. Neuropsychopharmacology. 2003;28(11):1973-1982. 